\title{
Knowledge of cardiovascular disease in South African HIV-positive surgical patients - A pilot survey
}

\section{Rachael Dellar,, Thandinkosi Enos Madiba" and} Yoshan Moodley ${ }^{\dagger}$

"Medical Sciences Department, University of Oxford, Oxford, United Kingdom

\#Department of Surgery, Nelson R. Mandela School of Medicine, University of KwaZulu-Natal, Durban, South Africa

tFaculty of Health \& Environmental Sciences, Central University of Technology, Bloemfontein, South Africa

\author{
Address for correspondence: \\ Yoshan Moodley \\ Faculty of Health \& Environmental Sciences \\ Central University of Technology \\ Private Bag X20539 \\ Bloemfontein \\ 9300 \\ South Africa
}

Email:

yoshan@hotmail.com

\section{INTRODUCTION}

South Africa is home to 7.1 million people living with HIV, representing almost 20\% of the global HIV-positive population. ${ }^{(1,2)}$ Access to antiretroviral therapy (ART) has improved in recent years; however, many of these individuals continue to suffer HIV-related complications. Often, such complications require surgical intervention, and HIV has now become a major contributor to the total burden of surgical disease in Africa. ${ }^{(3)}$ HIV mediates its impact on the surgical disease burden by promoting the development of multiple infections, inflammatory responses, and malignancies. ${ }^{(4)}$ Indeed, there have been increases in the incidence of numerous HIV-related conditions which require surgery, such as extra-pulmonary tuberculous, abscesses, and mucosal carcinoma. ${ }^{(5,6)}$ HIV prevalence is high in South African (SA) surgical patients. ${ }^{(7)}$ It is evident that medically treated HIV-positive patients are at risk for cardiovascular disease (CVD) due to the direct impact of HIV infection, or as a consequence of ART. However, a recent study demonstrated that the risk of peri-operative adverse cardiovascular events in younger HIV-positive surgical patients is similar to that observed in older, HIV-negative surgical patients.(8) Health promotion could play an important role in reducing CVD risk amongst the

\section{ABSTRACT}

Background: Medical and surgical HIV-positive patients are at risk of cardiovascular disease (CVD). Surgical patients are admitted to hospital for a few days around the time of their surgeries, allowing sufficient opportunity for health promotion interventions. Health promotion could improve CVD knowledge and encourage lifelong healthy behaviours. However, this approach requires that baseline CVD knowledge first be established. This study sought to determine the knowledge of CVD in HIV-positive South African (SA) surgical patients.

Methods: This was a prospective pilot survey of HIVpositive surgical patients who attended a tertiary hospital in Durban, South Africa, between I October 2016 and 31 March 2017. Patients completed 2 questionnaires: (I) a demographic characteristics questionnaire, and (2) a CVD knowledge questionnaire (identifying risk factors and signs/symptoms of myocardial infarction and stroke). All data were analysed using descriptive statistical methods.

Results: The study sample consisted of 39 HIV-positive surgical patients. Correct responses for the items on the CVD knowledge questionnaire ranged between $0.0 \%$ and $61.5 \%$ for risk factors, and between $0.0 \%$ and $\mathbf{8 9 . 7 \%}$ for signs/symptoms.

Conclusion: Knowledge of CVD risk factors and signs/ symptoms in this study was less than desirable. Levels of CVD knowledge are likely a function of educational attainment. SAHeart 2019;16:128-131

HIV-positive surgical population. Surgical patients are often hospitalised for several days around the time of their procedures, and this provides an opportunity to deliver health promotion activities in this patient group. Furthermore, the lessons learned through health promotion activities can instil positive behavioural changes which extend beyond the post-operative period, long after the patient has been discharged from hospital. However, effective planning of any such intervention requires establishing the baseline knowledge and understanding of CVD in this group. Therefore, the objective of this study was to explore the baseline knowledge of CVD risk factors and symptoms in a sample of HIV-positive surgical patients from Durban, South Africa. 


\section{MATERIALS AND METHODS}

Study setting and design

We conducted a prospective pilot survey of CVD knowledge in a population of $39 \mathrm{HIV}$-positive surgical patients attending the Inkosi Albert Luthuli Central Hospital in Durban, South Africa, between I October 2016 and 31 March 2017. The hospital is state-funded, and serves a population with a high HIV prevalence.

\section{Patients}

Adult HIV-positive patients from the surgical ward at the aforementioned hospital were approached to participate in this study. HIV status was determined following review of the patient medical record. These patients were given information leaflets about the research study, and given an opportunity to decide whether they wanted to participate in the research. Written informed consent was obtained from each patient who wished to participate in the research.

\section{Survey questionnaires}

Patients who consented to participation in this research were asked to complete two questionnaires. The first questionnaire collected basic demographic information, and the second questionnaire was related to CVD knowledge. The CVD knowledge questionnaire covered identification of risk factors and signs/ symptoms for myocardial infarction (MI) and stroke. The CVD knowledge questionnaire used in this study was adapted from questionnaires used in prior CVD knowledge surveys. ${ }^{(9,10)}$ Questionnaires for this study were made available in English and isiZulu languages. A bilingual research assistant was also present when patients were completing questionnaires to assist with further explanation of the questionnaires, if required.

\section{Statistical analysis}

All data were analysed using descriptive statistical methods. Results are presented as frequencies and percentages, or medians and interquartile ranges. The statistical analysis was performed using Stata version 13.0 (StataCorp, College Station, TX).

\section{Ethical approval}

This study was approved by the Biomedical Research Ethics Committee of the University of KwaZulu-Natal, Durban, South Africa (Protocol: BE498/16).

\section{RESULTS}

Patient demographics

The final study sample consisted of 39 patients. All patients in this study were of black African ethnicity. All patients were receiving ART. The median age of patients was 39.0 (interquartile range: 33.0 - 47.0) years. Most patients were female $(27 / 39,69.2 \%)$. The majority of patients had at least a secondary school education $(33 / 39,84.6 \%)$. Only a few patients (6/39, 15.4\%) had received prior education on CVD. Only 3/39 patients $(7.7 \%)$ had a prior personal medical history of CVD.

Patient knowledge of CVD risk factors

Table I shows the patient responses for the risk factor component of the CVD questionnaire. Correct identification of $\mathrm{MI}$ risk factors ranged between $0.0 \%$ and $61.5 \%$, depending on the risk factor. A similar range of correctly identified risk factors were observed for stroke. No patients were able to correctly identify non-white ethnicity and certain medications (including ART) as risk factors for MI. No patients could correctly identify age, family history of CVD, non-white ethnicity, excessive alcohol use, and lack of exercise as risk factors for stroke. Stress was the most commonly identified risk factor for both $\mathrm{Ml}$ and stroke.

\section{Knowledge of CVD signs/symptoms}

Table II shows the patient responses for the signs/symptoms component of the CVD questionnaire. Correct identification of $\mathrm{Ml}$ signs/symptoms ranged between $0.0 \%$ and $43.6 \%$, depending on the sign/symptom. Correct identification of

TABLE I: Knowledge of CVD Risk Factors.

\begin{tabular}{|l|c|c|}
\hline Risk factor & $\begin{array}{c}\text { Correctly } \\
\text { identified as } \\
\text { risk factor for } \\
\text { MI, } \mathbf{n}(\%)\end{array}$ & $\begin{array}{c}\text { Correctly } \\
\text { identified as } \\
\text { risk factor for } \\
\text { stroke, } \mathbf{n}(\%)\end{array}$ \\
\hline Older age & I (2.6) & $0(0.0)$ \\
\hline High cholesterol & $15(18.5)$ & I (2.6) \\
\hline High blood pressure & $3(7.7)$ & $7(18.0)$ \\
\hline Smoking & $4(10.3)$ & $1(2.6)$ \\
\hline Diabetes Mellitus & $8(20.5)$ & $7(18.0)$ \\
\hline Family history of CVD & $2(5.1)$ & $0(0.0)$ \\
\hline Non-white ethnicity & $0(0.0)$ & $0(0.0)$ \\
\hline Medications (ART) & $0(0.0)$ & $4(10.3)$ \\
\hline Obesity & $4(10.3)$ & $0(0.0)$ \\
\hline Unhealthy diet & $8(20.5)$ & $4(10.3)$ \\
\hline Excessive alcohol use & $3(7.7)$ & $0(0.0)$ \\
\hline Lack of exercise & $3(7.7)$ & $0(0.0)$ \\
\hline Stress & $24(61.5)$ & $24(61.5)$ \\
\hline \hline
\end{tabular}

CVD = cardiovascular disease, $A R T=$ antiretroviral therapy. 
TABLE II: Knowledge of CVD signs/symptoms.

\begin{tabular}{|c|c|c|c|}
\hline Sign/symptom of MI & Correctly identified, $\mathrm{n}(\%)$ & Sign/symptom of stroke & Correctly identified, $n$ (\%) \\
\hline Chest pain & $6(15.4)$ & Numbness on one side of the body & $35(89.7)$ \\
\hline Shortness of breath & $17(43.6)$ & Facial weakness & $22(56.4 I)$ \\
\hline Heavy chest & $9(23.1)$ & Visual disturbance & I (2.6) \\
\hline Dizziness & $0(0.0)$ & Confusion & $2(5.1)$ \\
\hline Sweating & $7(18.0)$ & High blood pressure & $0(0.0)$ \\
\hline Nausea/vomiting & $3(7.7)$ & Slurred speech & $9(23.1)$ \\
\hline Feeling light-headed & $0(0.0)$ & Weakness on one side of the body & $15(38.5)$ \\
\hline Back pain & I (2.6) & Severe headache & $0(0.0)$ \\
\hline Upset stomach & I (2.6) & & \\
\hline
\end{tabular}

CVD = cardiovascular disease, $\mathrm{Ml}=$ myocardial infarction

stroke signs/symptoms ranged between $0.0 \%$ and $89.7 \%$, depending on the sign/symptom. No patients were able to correctly identify dizziness and feeling light-headed as signs/ symptoms of Ml. No patients could correctly identify high blood pressure and severe headache as signs/symptoms of stroke. Shortness of breath was the most commonly identified Ml sign/ symptom. Numbness on one side of the body was the most commonly identified stroke sign/symptom.

\section{DISCUSSION}

Overall, our study findings suggest that CVD knowledge in SA HIV-positive surgical patients requires substantial improvement. While there were several CVD risk factors and signs/symptoms which $<10 \%$ of patients in this study could correctly identify, there were several CVD risk factors and signs/symptoms which no patients could correctly identify.

One of the most popular models used in health promotion, the Health Belief Model (HBM), consists of several key concepts including: perceived susceptibility, perceived severity, perceived benefits of health-promoting behaviour, perceived barriers to health-promoting behaviour, cues to action, and self-efficacy. ${ }^{(1)}$ Knowledge of a disease condition can directly influence an individual's perceived susceptibility/severity of a disease, perceived barriers/benefits to health-promoting behaviour, and the perceived threat of disease. Perceived susceptibility/severity and cues to action can directly influence the perceived threat of disease. The perceived threat of disease, and the weighing of benefits and barriers related to health-promoting activities, determines the likelihood of an individual engaging in these activities. (II) In the context of this study, HIV-positive surgical patients did not identify medication use (including long-term ART) or non-white ethnicity as risk factors for MI. However, the literature suggests that these are indeed risk factors for MI. ${ }^{(12,13)}$ These misconceptions could lead to lower perceived susceptibility and threat for $\mathrm{Ml}$ in these patients, and these patients might ultimately be less likely to engage in CVD screening. In addition, excessive alcohol use is often described in the literature as a risk factor for stroke, ${ }^{(14,15)}$ but was not identified as a stroke risk factor by any patients in this study. Patients who are oblivious to the CVD risk associated with alcohol might continue to consume large amounts of alcohol.

Knowledge of common signs and symptoms of CVD was poor in this study. In many patients, signs and symptoms of CVD usually evolve over a period of time. For instance, a patient may experience angina for several weeks before suffering MI.(16) Transient ischaemic attack shares several signs and symptoms with stroke, ${ }^{(17)}$ many of which were not correctly identified by the study population. If patients have no knowledge or incorrect knowledge of CVD signs and symptoms, they might be less likely to seek CVD screening or related healthcare services at an earlier stage of the disease process when these signs and symptoms first appear.(18,19) Unfortunately, these patients may only seek CVD screening services when it is too late and the CVD has reached an advanced stage where there is high morbidity and mortality. Therefore, the importance of appropriate CVD knowledge in at-risk populations also extends to signs and symptoms.

This study was not without limitations. The sample size was very small. The small sample size did not allow for an exploration of statistical associations between demographic characteristics 
and CVD knowledge. Additionally, this was a single-centre study with a geographically and ethnically homogeneous sample: young, black, HIV-positive patients with mostly low levels of education. It is therefore impossible to generalise the findings of this study to the entire population of HIV-infected persons in South Africa. Only patients with a record of a positive HIV test in their medical record were approached to participate in this survey. Therefore, patients who might have been HIV-positive but did not have this reported in their medical record might not have been approached to participate in this study. We did not collect data on patient residence (rural or urban), and we could not investigate the impact of this specific variable on CVD knowledge in this study. There was no HIV-negative control group in this study. We recommend that future research on this topic in our setting also include an HIV-negative control group for comparative analysis. We also recommend that these HIV-positive and HIV-negative groups be matched on age, sex, ethnicity, educational attainment and urban/rural residence to adjust for the potentially confounding influence of these variables in a comparative analysis between the 2 groups. Lastly, patients in this study were not questioned about their perceived risk of CVD.

In conclusion, knowledge of CVD risk factors and signs/symptoms in this study was less than desirable. Levels of CVD knowledge observed in this study are likely a function of educational attainment.

\section{Conflict of interest: none declared.}

\section{REFERENCES}

I. Murray CJ, Ortblad KF, Guinovart C, et al. Global, regional, and national incidence and mortality for HIV, tuberculosis, and malaria during 1990-20 I3: A systematic analysis for the Global Burden of Disease Study 2013. Lancet 2014:384:1005-70.

2. UNAIDS. (2018) South Africa 2017: Country fact sheet [online]. Retrieved August 28, 2018: http://www.unaids.org/en/regionscountries/countries/ southafrica.

3. Odimba BFK. The impact of HIV infection on the surgical disease burden in Africa. East Cent Afr J Surg 20 I0; 15:3-8.

4. Bowa K, Kawimbe B, Mugala D, et al. A Review of HIV and surgery in Africa. Open AIDS J 20 I6; 10:16-23.

5. Kalyesubula R, Wearne N, Semitala FC, Bowa K. HIV-associated renal and genitourinary comorbidities in Africa. J Acquir Immune Defic Syndr 20 I 4;67 Suppl I:S68-78.

6. Dua RS, Wajed SA, Winslet MC. Impact of HIV and AIDS on surgical practice. Ann R Coll Surg Engl 2007:89:354-58.

7. Cacala SR, Mafana E, Thomson SR, Smith A. Prevalence of HIV status and CD4 counts in a surgical cohort: Their relationship to clinical outcome. Ann R Coll Surg Engl 2006;88:46-5I.

8. Redman LA, Naidoo P, Biccard BM. HIV, vascular surgery and cardiovascular outcomes: A South African cohort study. Anaesthesia 2014;69:208-13.

9. Hickey A, Holly D, McGee H, Conroy R, Shelley E. Knowledge of stroke risk factors and warning signs in Ireland: Development and application of the Stroke Awareness Questionnaire (SAQ). Int J Stroke 20 I2;7:298-306.

10. Prendergast HM, Bunney EB, Roberson T, Davis T. Knowledge of heart disease among women in an urban emergency setting. J Natl Med Assoc 2004;96: 1027-31.

II. Twente University (2017). Health communication - Health Belief Model [online]. Retrieved August 28, 2018: https://www.utwente.nl/en/bms/ communication-theories/sorted-by-cluster/Health\%20Communication/ Health_Belief_Model/.

12. Thienemann F, Sliwa K, Rockstroh JK. HIV and the heart: The impact of antiretroviral therapy: A global perspective. Eur Heart J 20 I 3;34:3538-46.

13. Ferdinand KC, Yadav K, Nasser SA, et al. Disparities in hypertension and cardiovascular disease in blacks: The critical role of medication adherence. J Clin Hypertens (Greenwich) 2017;19:1015-24.

14. Daniel S, Bereczki D. Alcohol as a risk factor for haemorrhagic stroke. Ideggyogy Sz 2004;57:247-56.

15. Larsson SC, Wallin A, Wolk A, Markus HS. Differing association of alcohol consumption with different stroke types: A systematic review and metaanalysis. BMC Med 2016; 14:178.

16. Harper RW, Kennedy G, DeSanctis RW, Hutter AM, Jr. The incidence and pattern of angina prior to acute myocardial infarction: A study of 577 cases. Am Heart J 1979;97: 178-83.

17. Nadarajan V, Perry RJ, Johnson J, Werring DJ. Transient ischaemic attacks: Mimics and chameleons. Pract Neurol 20|4;14:23-31.

18. Mellor RM, Bailey S, Sheppard J, et al. Decisions and delays within stroke patients' route to the hospital: A qualitative study. Ann Emerg Med 2015;65:279-87.e273.

19. Whitaker S, Baldwin T, Tahir M, Choudhry O, Senior A, Greenfield S. Public knowledge of the symptoms of myocardial infarction: A street survey in Birmingham, England. Fam Pract 2012;29:168-73. 